\title{
MULTI-PHYSICAL AND MULTI-SCALE DETERIORATION MODELLING OF RE- INFORCED CONCRETE: MODELLING CORROSION-INDUCED CONCRETE DAMAGE
}

\author{
A. MICHEL ${ }^{*}$, M. LEPECH ${ }^{\dagger}$, H. STANG ${ }^{\dagger \dagger}$, AND M.R. GEIKER ${ }^{\dagger+\dagger}$ \\ Technical University of Denmark \\ Kgs. Lyngby, Denmark \\ e-mail: almic@byg.dtu.dk \\ Stanford University \\ Stanford, California, USA \\ e-mail: mlepech@stanford.edu \\ ${ }^{\dagger \dagger}$ Technical University of Denmark \\ Kgs. Lyngby, Denmark \\ e-mail: hs@byg.dtu.dk \\ ${ }^{\dagger \dagger}$ Norwegian University of Science and Technology \\ Trondheim, Norway \\ e-mail: mette.geiker@ntnu.no
}

Key words: Multi-physics, Multi-scale, Deterioration, Lattice, FEM, Fracture

\begin{abstract}
In this paper, corrosion-induced damage is investigated by means of numerical simulations utilizing a coupled lattice and finite element method (FEM) modelling approach. While the reinforcement and corrosion product domain are discretized by the FEM, a lattice approach is used for the discretization of the concrete domain. To model the expansive nature of solid corrosion products, a thermal analogy is used. The modelling approach further accounts for the penetration of solid corrosion products into the available pore space of the surrounding cementitious materials and non-uniform distribution of corrosion products along the circumference of the reinforcement. Finally, results of numerical simulations in terms of corrosion-induced deformations and cracking are compared to experimental data obtained by digital image correlation and published in the literature.
\end{abstract}

\section{INTRODUCTION}

Deterioration of civil infrastructure (bridges, tunnels, roads, and buildings) together with increasing functional requirements (e.g. traffic load and intensity) present major challenges to society in most developed countries. A major part of the infrastructure is built from reinforced concrete. The costs for maintenance, renovation, and renewing are growing and now take up a major part of concrete infra- structure investments. While engineering tools and methods are well developed for the structural design of infrastructures, tools for assessing the current and predicting the future condition of reinforced concrete structures are less advanced. Existing prediction tools are largely empirical, and thus limited in their ability to predict the performance of new materials, structural, or maintenance solutions. As such, the inability to reliably assess the long- 
term future ramifications of today's design decisions poses a major obstacle for the design of reinforced concrete structures. A primary reason for the lack of reliable modelling tools is that deterioration mechanisms are highly complex, involve numerous coupled physical phenomena that must be evaluated across a range of scales, and often cut across several academic disciplines and faculties.

In this paper, a multi-physics and multiscale modelling approach for deterioration of a reinforced concrete structure is presented. Focus is, thereby, placed on a modelling approach for corrosion-induced damage, which is embedded in an overarching cross-disciplinary numerical framework that combines mechanical, chemical, and electrochemical processes on different length scales in reinforced concrete. Corrosion-induced damage processes are investigated by means of numerical simulations utilizing a coupled lattice and finite element method (FEM) modelling approach. While the domains for reinforcement and corrosion products are discretized by the FEM, a lattice approach is used for the discretization of the concrete domain. To model the expansive nature of solid corrosion products, a ther- mal analogy is used. Moreover, the modelling approach accounts for the penetration of solid corrosion products into the available pore space of the surrounding cementitious materials as well as non-uniform distribution of corrosion products along the circumference of the reinforcement. Finally, numerical simulations and experimental observations (obtained by digital image correlation and published in the literature), i.e. corrosion-induced deformations and cracking, are compared.

\section{LINKING MULTIPLE SCALES AND DETERIORATION PROCESSES IN RE- INFORCED CONCRETE}

The overarching multi-physics and multiscale modelling framework is presented in Fig. 1 , describing (a) transport of heat and matter and chemical processes resulting in changes in phase assemblage in hydrated Portland cement, $(b)$ electrochemical processes at the reinforcement surface, $(c)$ material performance including corrosion-induced damages on the material and meso-scale, and $(d)$ structural deterioration of reinforced concrete components due to reinforcement corrosion. Individual science-based models, describing actual

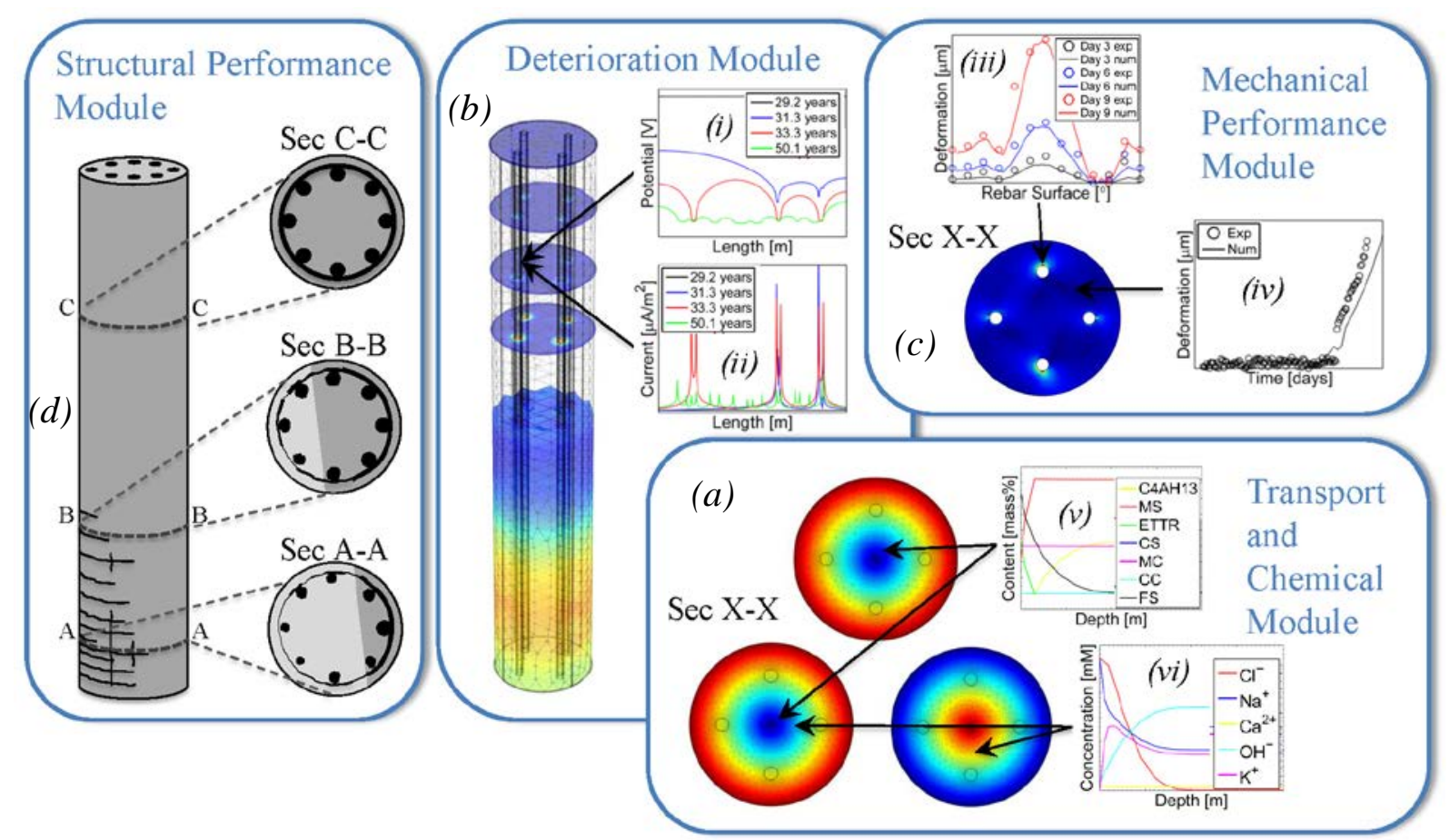

Figure 1: Multi-physics and multi-scale modelling framework for deterioration of reinforced concrete including physical, chemical, electrochemical, and fracture mechanical processes at the material and meso-scale, which are further coupled with mechanical deterioration processes at the structural/component scale, from [1]. 
physical phenomena, are organized into modules in this modelling framework. To establish a link between the individual modules, an interface module allows for passing of information from one module to another, thereby bridging various length and time scales and allowing for concurrent performance simulations of reinforced concrete structures.

The framework is fully coupled in the sense that information, such as phase assemblage, moisture distribution, corrosion rate, damage state of concrete cover, etc., are continuously exchanged between the modules. More detailed information on the theoretical background and implementation of the various modules in the multi-physics and multi-scale modelling framework are given in e.g. [1-5].

\section{CORROSION-INDUCED CONRETE DAMAGE}

Once corrosion is initiated, electrochemical half-cell reactions are taking place along the reinforcement, see also Fig. 1 - 'Deterioration Module'. The ionic reaction products of those half-cell reactions may further react and form solid corrosion products in the vicinity of the reinforcement. The type of corrosion products formed depends on the thermodynamic conditions present in the vicinity of the reinforcement [6]. Apart from solid corrosion products, soluble iron-chloride complexes (also referred to as green rust) may form in an oxygendeprived environment in which chlorides are present [6,7]. Such soluble iron-chloride complexes may not necessarily form in the vicinity of the reinforcement surface, as shown e.g. in [6]. The location and formation of solid corrosion products along the circumference of the reinforcement can be directly obtained from the 'Deterioration Module' (see Fig. 1) within the multi-physics and multi-scale modelling framework.

Independent of the type of iron oxides formed as a result of active corrosion, iron oxides occupy a larger volume than the initial iron that is consumed during the corrosion reaction, see e.g. [8]. The increased volume of corrosion products causes tensile stresses in the surrounding concrete and may lead to con- crete cracking, spalling, or delamination if the tensile strength of the concrete is exceeded.

\subsection{Modelling approach}

To model the expansive nature of solid corrosion products, a thermal analogy is used in which a 'fictitious' thermal load is applied to the corroded reinforcement section (see Fig. 2) to mimic the increased volume of corrosion products. Basic geometrical considerations and methodology of load application in the coupled lattice and FEM model are illustrated in Fig. 2. For the determination of the corroded reinforcement section (see Fig. 2), Faraday's law is used, which describes the relation between thickness reduction per time and corrosion current density, which can be predicted by the 'Deterioration Module' (see Fig. 1)

$$
\mathrm{X}(t)=\frac{M_{F e}}{z F_{a} \rho_{F e}} \int_{0}^{t} i_{c o r r}(t) d t
$$

where $X(t)$ is the cross sectional reduction of the reinforcement as a function of time, $t$, $M_{\mathrm{Fe}}$ the molar mass of iron, $\mathrm{z}$ the number of electrons transferred in the half-cell reaction, $F_{a}$ Faraday's constant, and $\rho_{F e}$ the density of iron. Assuming a constant coefficient of thermal expansion, $\alpha$, the applied temperature increment, $\Delta T$, represents then the type of solid corrosion product (see Fig. 2). Assuming further isotropic material properties of the corrosion products, the linear expansion coefficient, $\eta_{\text {lin }}$, may be obtained as one third of the volume expansion coefficient.

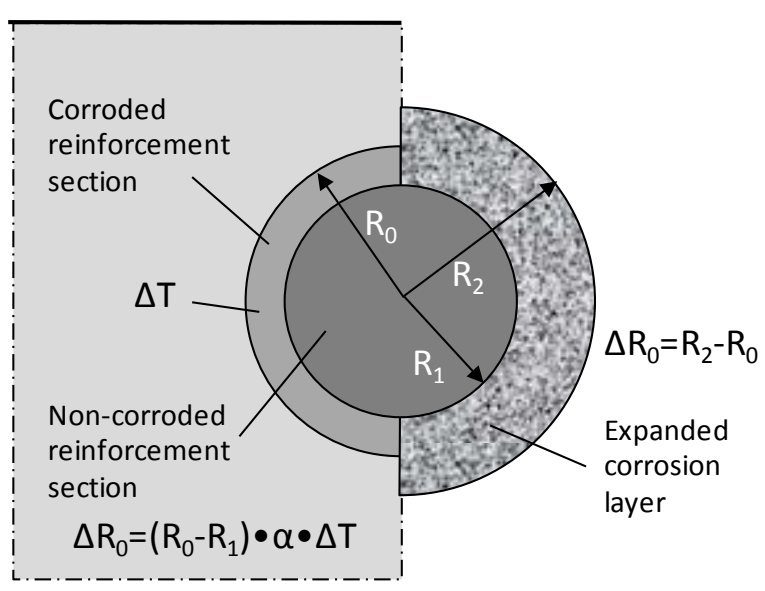

Figure 2: Load application and geometrical considerations in lattice/FEM model to simulate corrosioninduced damage, from [9]. 


\subsection{Coupled lattice and FEM model}

Various models dealing with fracture processes in quasi-brittle materials, such as concrete, have been developed. In particular, discrete and smeared cracking approaches, see e.g. $[10,11]$, gained popularity in efforts to reproduce experimentally observed fracture characteristics of concrete. However, both smeared and discrete cracking approaches lack description of fracture processes in detail, such as formation of micro-cracks, crack branching, etc. The application of lattice fracture models provides an opportunity to compensate for the drawbacks of discrete and smeared cracking approaches. In the last two decades, large efforts have been made to develop and study lattice models and the effects of regular and irregular lattice networks e.g. [12,13], use of triangular and quadrangular meshes e.g. $[14,15]$, use of truss and beam elements e.g. $[13,16]$, and implementation of heterogeneity with respect to the fracture process e.g. [14].

Within the present approach a random, triangular lattice of truss elements is chosen to investigate corrosion-induced damage in concrete. As the formation of cracks is assumed to take place only in the concrete domain, the remaining domains (steel and corrosion layer) are modelled with continuum elements. The random lattice of truss elements in the concrete domain is established by initially creating a regular square lattice in the concrete domain. Within each cell of the regular lattice a point is selected at random with uniform distribution. Subsequently, Voronoi construction is used to create a random lattice by connecting the three points that are closest to each other. The randomness of the lattice can be varied through the introduction of a sub-cell in the square lattice, from which the random point is chosen.

Crack formation and growth within the concrete domain is achieved by removing the element with the highest stress to tensile strength ratio and greater than unity. Upon the next loading step the updated system of equations is recomputed and the analysis repeated until the system fails. This step-by-step removal of lattice elements allows crack evolution in the specimen and for tracking of mi- crocrack propagation and crack pattern in the simulation. To determine the nodal displacements and subsequently the stresses in the elements of the lattice for each analysis step an elastic analysis is performed.

In general, 2-D nodal displacements can be obtained as follows for a set of linear equations within a FEM formulation.

$$
\{F\}=[K]\{U\}
$$

where $F$ is the global nodal force vector, $K$ the global stiffness matrix, and $U$ the global nodal displacement vector. The global stiffness matrix is hereby obtained as follows.

$$
[K]=\int\left[B^{T}\right][D][B] h d \Omega
$$

where $B$ is the strain matrix, $D$ the constitutive material matrix, and $h$ the thickness of the domain, $\Omega$. In the present modelling approach, lattice and continuum elements are combined to discretize the various domains of the model, i.e. the concrete domain is discretized using truss elements while the corrosion product and reinforcement domain are discretized using bilinear quadrilateral and linear triangular elements, respectively.

The element stiffness matrix for the truss elements of the lattice can be obtained as follows.

$$
K_{c}=\frac{E_{c} A_{c}}{L_{c}}\left[\begin{array}{cc}
k_{c 11} & -k_{c 11} \\
-k_{c 11} & k_{c 11}
\end{array}\right]
$$

where $E_{c}$ is the concrete stiffness, $A_{c}$ the cross sectional area, $L_{c}$ the length, and $k_{c 11}$ is

$$
k_{c 11}=\left[\begin{array}{cc}
\cos \theta^{2} & \cos \theta \sin \theta \\
\cos \theta \sin \theta & \sin \theta^{2}
\end{array}\right]
$$

where $\theta$ is angle between the positive global $\mathrm{x}$-axis and the corresponding truss element.

For linear triangular elements in the steel domain, the element stiffness matrix is defined as follows.

$$
\left[K_{s}\right]=\left[B_{s}^{T}\right]\left[D_{s}\right]\left[B_{s}\right] h\left\{A_{s}\right\}
$$

where $A_{s}$ is the element area and $B_{s}$ is

$$
\left[B_{s}\right]=\left[\begin{array}{lll}
B_{s i} & B_{s j} & B_{s m}
\end{array}\right]
$$

with 


$$
\left[B_{\text {si..m }}\right]=\frac{1}{2 A_{s}}\left[\begin{array}{cc}
\beta_{i . . m} & 0 \\
0 & \gamma_{i . . m} \\
\gamma_{i . . m} & \beta_{i . . m}
\end{array}\right]
$$

with

$$
\beta_{i . . m}=y_{j . . i}-y_{m . . j}
$$

and

$$
\gamma_{i . . m}=x_{j . . i}-x_{m . . j}
$$

where $x$ and $y$ describe coordinates of the $i^{\text {th }}, j^{\text {th }}$, and $m^{\text {th }}$ node of the triangular element.

Finally, the element stiffness matrix for the bilinear quadrilateral elements discretizing the corrosion product domain can be written as follows.

$$
\left[K_{c p}\right]=\int_{A_{c p}}\left[B_{c p}^{T}\right]\left[D_{c p}\right]\left[B_{c p}\right] h d A_{c p}
$$

The integration over the element can be evaluated using Gauss quadrature as follows.

$$
=h \sum_{i=1}^{n g p} \sum_{j=1}^{n g p} w_{i} w_{j}\left[B_{c p}^{T}\right]\left[D_{c p}\right]\left[B_{c p}\right] \operatorname{det}[J]
$$

where $w_{i}$ and $w_{j}$ are Gauss weights and $\operatorname{det}[J]$ is the determinant of the Jacobian. An exact solution of the integral is obtained for at least two Gauss points in each direction. The strain matrix $B_{c p}$ can be defined as follows.

$$
\left[B_{c p}\right]=\left[\begin{array}{llll}
B_{c p i} & B_{c p j} & B_{c p m} & B_{c p n}
\end{array}\right]
$$

with

$$
\left[B_{c p i . . n}\right]=\left[\begin{array}{cc}
\frac{\partial N_{i . . n}}{\partial x} & 0 \\
0 & \frac{\partial N_{i . . n}}{\partial y} \\
\frac{\partial N_{i . . n}}{\partial y} & \frac{\partial N_{i . . n}}{\partial x}
\end{array}\right]
$$

where $N_{i . . n}$ are shape functions of the bilinear quadrilateral elements with nodes $i, j, m$, and $n$ in natural coordinates as follows.

$$
N_{i . . n}(\xi, \eta)=0.25(1-\xi-\eta-\xi \eta)
$$

The thermal analogy to mimic the expansive nature of corrosion products (see also Fig.
2 ) is realized by equivalent nodal forces, $f_{c p, t}$, for the corrosion product domain, which can be determined as follows.

$$
\left[f_{c p, t}\right]=\int_{A_{c p}}\left[B_{c p}^{T}\right]\left[D_{c p}\right]\left[\varepsilon_{c p, t}\right] h d A_{c p}
$$

The thermal strain matrix, $\varepsilon_{c p, t}$, is given as follows for two dimensional problems

$$
\left[\varepsilon_{c p, t}\right]=\left[\begin{array}{c}
\alpha \Delta T \\
\alpha \Delta T \\
0
\end{array}\right]
$$

Using Gauss quadrature, the integration over the element of the corrosion product domain (Eq. 16) can be evaluated as follows.

$$
=h \sum_{i=1}^{n g p} \sum_{j=1}^{n g p} w_{i} w_{j}\left[B_{c p, t}^{T}\right]\left[D_{c p}\right]\left[\varepsilon_{c p, t}\right] \operatorname{det}[J]
$$

The modelling approach is formulated in Matlab $^{\circledR}$, and focus was placed on efficient algorithms to allow for simulations with large numbers of degrees of freedom (DOFs), i.e. tens of thousands of elements. In particular, the sparse command in Matlab ${ }^{\circledR}$ allows for simple assemblage of the global stiffness matrix as a sum of the individual domain stiffness matrices, i.e.

$$
[K]=\left[K_{c}\right]+\left[K_{s}\right]+\left[K_{c p}\right]
$$

To solve the set of linear equations (see Eq. 2) and ensure convergence of the solution, an iterative conjugate gradients squared method solver is used. Finally, it should be noted that the simulation of the corrosion process is time dependent, i.e. the corrosion product domain is growing. This requires reassembly of the corrosion product and steel domain for every 'time step'. To account for the corrosioninduced damage from one 'time step' to another, an internal nodal force vector is created for each time step, which assures that corrosion-induced damage is transferred from the previous 'time step'. The internal nodal force vector, $f_{i}$, can be calculated as follows.

$$
\left[f_{i}\right]=\int_{A}\left[B^{T}\right][\sigma] h d A
$$

where $\sigma$ is the element stress. 
To verify the presented modelling approach, results of the coupled lattice and FEM model are compared to results of the commercial FEM software COMSOL Multiphysics ${ }^{\circledR}$. In the example, a square concrete domain with a centered rebar (radius of $5 \mathrm{~mm}$ ) surrounded by a uniform corrosion product domain is modelled. The corrosion product domain is subsequently exposed to a 'fictitious' temperature increment, mimicking the expansive nature of corrosion products. No elements are removed during the solution of the coupled lattice and FEM model. The geometry and material properties of the individual domains for the example are illustrated in Fig. 3. All corners of the concrete domain are fixed in both $\mathrm{x}$ and $\mathrm{y}$ directions.

Comparisons between corrosion-induced deformations along the circumference of the reinforcement obtained from the commercial FEM tool and the presented coupled lattice and FEM model for the chosen example are given in Figs. 4 and 5. As can be seen from the results, the number of elements used to discretize the interface between corrosion product and concrete domain has an influence on the corrosion-induced deformations in the concrete domain along circumference of the rebar. Very good agreements between the commercial FEM tool and proposed modelling approach are obtained once the interface is discretized with more than 36 elements.

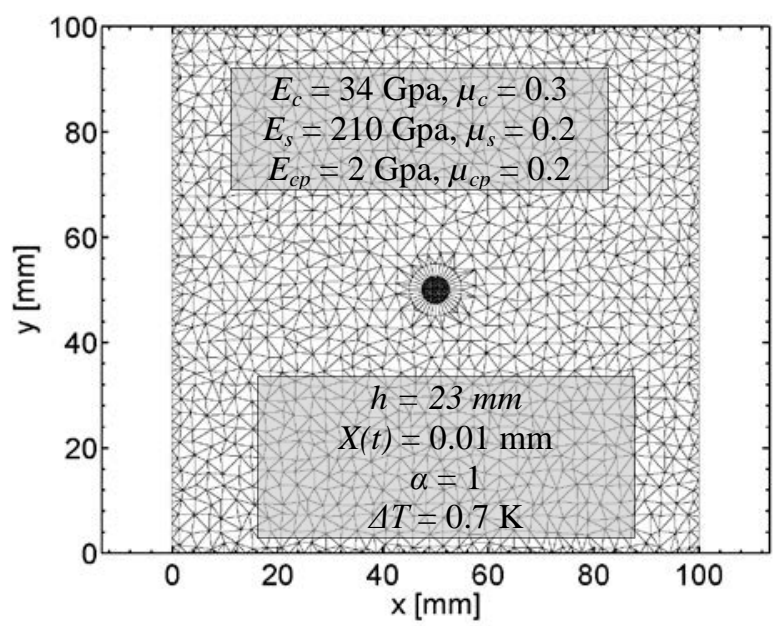

Figure 3: Geometry and material properties for example to compare commercial FEM and coupled lattice and FEM models. Note: corrosion product domain magnified by factor 500 for visibility.
No significant influence of the number of elements in the concrete domain on the corrosion-induced deformation along the circumference of the reinforcement is observed, as illustrated in Fig. 5.

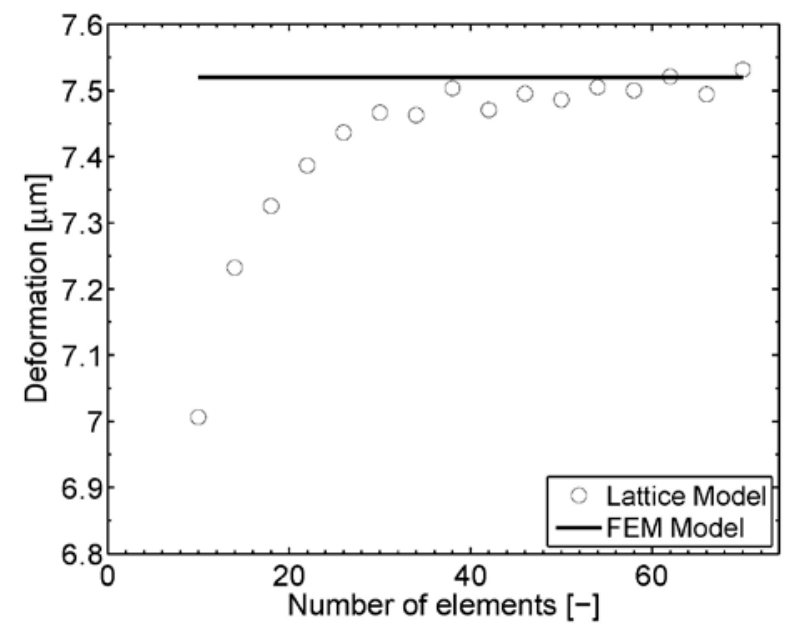

Figure 4: Influence of element number along the circumference of the reinforcement on corrosioninduced concrete deformations.

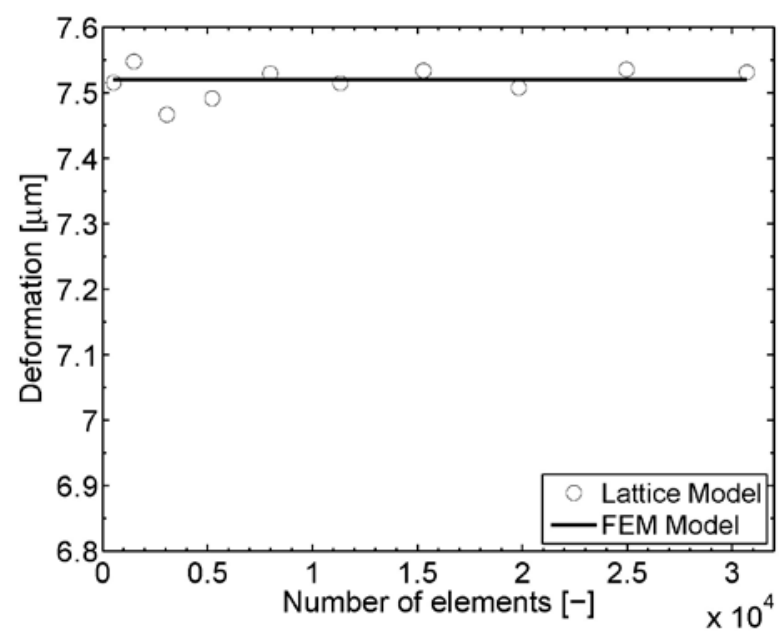

Figure 5: Influence of element number in concrete domain on corrosion-induced concrete deformations.

\subsection{Penetration of corrosion products into concrete}

Experimental and numerical investigations, see e.g. [17-20], suggest that part of the corrosion products penetrate into open pore space/voids of the surrounding concrete delaying corrosion-induced stress formation, see Fig. 6.

Based on the experimental results presented in [20] a conceptual model was established in 
[21] describing the penetration of corrosion products into the available pore space of cementitious materials surrounding corroding reinforcement. In [21], it is assumed that an initial corrosion accommodating region $(C A R)$ around the reinforcement exists, denoted $C A R_{0}$, which delays stress formation while filling with solid corrosion products, see Fig. 7. Once this initial $C A R_{0}$ is filled with corrosion products, tensile stresses in the surrounding cementitious material increase and potentially lead to the formation of micro-cracks. These microcracks allow solid corrosion products to penetrate additional pore spaces and further delay corrosion-induced stresses. At some point a maximum size of the $C A R$, denoted as $C A R_{M A X}$, is reached. No corrosion products can penetrate the matrix of the cementitious material beyond that point and all additionally formed corrosion products will introduce tensile stresses and potentially lead to the formation of a macrocrack.

The accommodation of corrosion products in the $C A R$ may be accounted for by an adjusted temperature increment, $\Delta T_{C A R}$, in the coupled lattice and FEM model (see Eq. 17)

$$
\Delta T_{C A R}=\lambda_{C A R} \Delta T
$$

where $\lambda_{C A R}$ describes the penetration of corrosion products into the accessible pore space of the cementitious material, $V_{C A R}$, and may be defined as shown in Eq. 22.

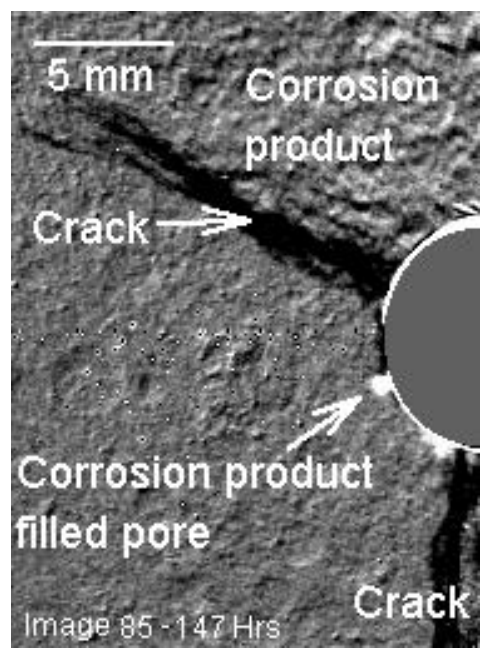

Figure 6: X-ray attenuation measurement image of corrosion products and subsequent concrete cracking after 147 hours of accelerated corrosion testing, from [20].

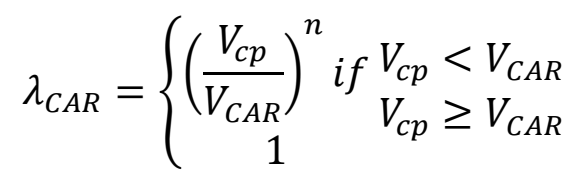

where $n$ is a non-physical fitting parameter (a value of 1.3 for $n$ was found to provide the best fit to the experimental data in [21]) and $V_{c p}$ the expanded volume of corrosion products

$$
V_{c p}=\eta_{v o l} \pi h\left(r^{2}-(r-X(t))^{2}\right)
$$

where $\eta_{v o l}$ is the dimensionless volumetric expansion coefficient of corrosion products and $X(t)$ the rebar radius reduction. Assuming the $C A R$ consists of the capillary pores of the cementitious material, $\varphi$, the $C A R$ volume, $V_{C A R}$, may be determined as follows.

$$
V_{C A R}=\varphi V_{c m}
$$

where the capillary porosity may be approximated using Powers’ law [22].

$$
\varphi=\frac{w / c-0.36 \chi}{w / c+0.32}
$$

where $\chi$ is the degree of hydration. The accessible volume of the cementitious matrix, $V_{c m}$, which is controlled by the size of the $C A R$, may be determined as follows.

$$
V_{c m}=\pi h\left((r+C A R)^{2}-r^{2}\right)
$$

where $r$ is the radius of the rebar and the $C A R$ can be described as follows.

$$
C A R=C A R_{0}+\left(C A R_{M A X}-C A R_{0}\right) \kappa
$$

where $\kappa$ is a dimensionless coefficient describing the change in connectivity of accessible pore space inside the CAR. $\kappa$ is assumed to vary between 0 and 1 according to

$$
\kappa=\left\{\begin{array}{lc}
0 & V_{c p} \leq V_{c p, \min } \\
\delta \text { if } & V_{c p, \min }<V_{c p} \leq V_{c p, \max } \\
1 & V_{c p}>V_{c p, \max }
\end{array}\right.
$$

with $\delta$

$$
\delta=\frac{V_{c p}-V_{c p, \min }}{V_{c p, \max }-V_{c p, \min }}
$$

where $V_{c p \text { min }}$ is the minimum volume of corrosion products and $V_{c p \text {, max }}$ the maximum volume of corrosion products that can be accommodated in the $C A R$. 


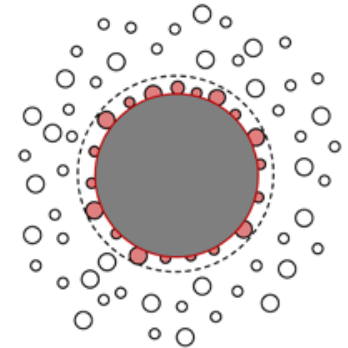

(a)

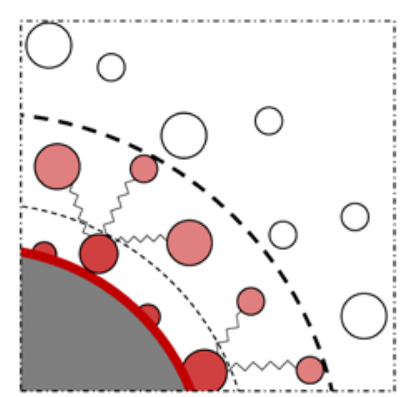

(c)

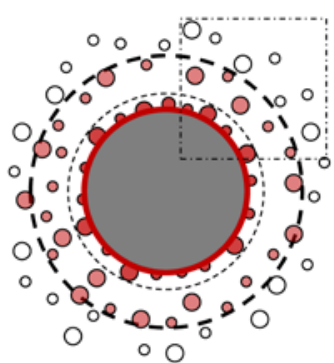

(b)

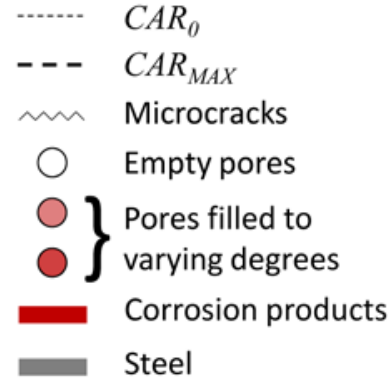

Steel
Figure 7: Conceptual schematic of idealized filling process of capillary porosity with corrosion products: (a) shows the initial $C A R, C A R_{0}$, (b) the subsequent increase in $C A R$ size to a maximum, $C A R_{M A X}$ and filling of additional pores due to (c) formation of microcracks between pores allowing movement of corrosion products, from [21].

\subsection{Non-uniform reinforcement corrosion}

Experimental observations presented in $[20,21]$ also suggest non-uniform deformations along the circumference of the reinforcement, which indicates non-uniform deposition of corrosion products. The non-uniform deposition of corrosion products can be explicitly accounted for in the coupled lattice and FEM model by varying the corrosion current density (see Eq. 1) along the circumference of the reinforcement and thereby generating different degrees of corrosion of the reinforcement.

\section{COMPARISON OF EXPERIMENTAL AND NUMERICAL RESULTS}

To test the ability of the proposed model to simulate and predict corrosion-induced deformations and crack formation, numerical results are compared to experimental observations presented in $[21,23]$. In the experiment, a $23 \times 100 \times 100 \mathrm{~mm}^{3}$ reinforced mortar specimen was subjected to accelerated corrosion by an impressed electrical current of $100 \mu \mathrm{A} / \mathrm{cm}^{2}$.

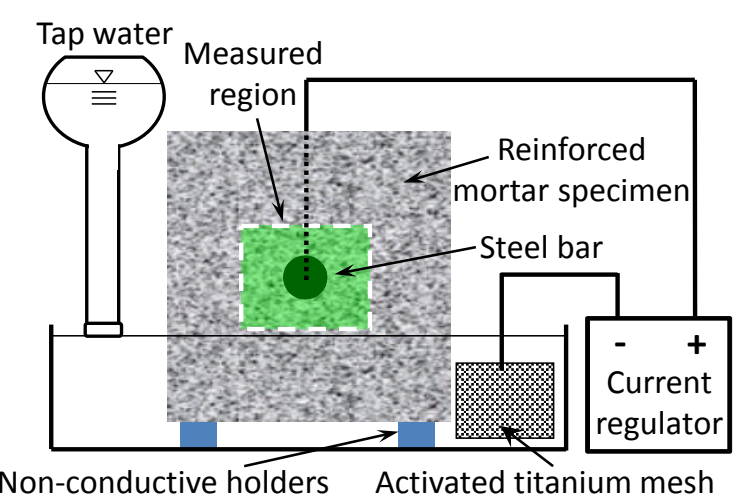

Figure 8: Experimental setup for accelerated corrosion by impressed current, 'measured region' indicating area captured in digital images, from [23].

One smooth $10 \mathrm{~mm}$ steel reinforcement bar was embedded in the center of the mortar specimen with w/c ratio of 0.5 . To provide an electrical connection between working (reinforcement) and counter electrode (ruthenium/iridium electrode), the specimen was placed in tap water, which was maintained at a level of approximately $10 \mathrm{~mm}$ below the reinforcement, see Fig. 8. Corrosion-induced deformations and crack formation were observed by means of digital image correlation (DIC) and the experiment was stopped once the first macrocrack was observed. For more detailed information about the experimental setup reference is made in $[21,23]$.

The input parameters for the numerical simulation are given in Table 1 . The model consisted of 83,972 DOFs, with 122,467 lattice elements discretizing the concrete domain, 100 bilinear quadrilateral and 1092 linear triangular elements were used to discretize the corrosion product and reinforcement domain, respectively. Within the concrete domain the lattice element length varied between approximately 9 and $800 \mu \mathrm{m}$.

The linear expansion coefficient, $\eta_{\text {lin }}$, was set to 0.7 assuming the formation of hematite $\left(\mathrm{Fe}_{2} \mathrm{O}_{3}\right)$. The 'fictitious' thermal expansion coefficient, $\alpha$ (see Eq. 17), was set constant, i.e. 1, while the adjusted temperature increment, $\Delta T_{C A R}$ (see Eq. 21), accounted for the penetration and non-uniform deposition of corrosion products. The experimental data was fitted adjusting the impressed corrosion current density and thus the deposition of corrosion products along the circumference of the 
reinforcement. As a starting point for the estimation of the impressed corrosion current density, DIC measured deformations after three days of accelerated corrosion were used. Results of the presented modelling approach and experimental observations, i.e. corrosioninduced deformations along the circumference of the reinforcement, are given in Fig. 9.

Table 1: Input parameters for coupled lattice and FEM model to simulate experimental observations.

\begin{tabular}{ccc}
\hline Parameter & Value & Unit \\
\hline$M_{F e}$ & 55.845 & {$[\mathrm{~g} / \mathrm{mol}]$} \\
\hline$z$ & 2 & {$[-]$} \\
\hline$\rho_{F e}$ & 7.86 & {$\left[\mathrm{~g} / \mathrm{mm}^{3}\right]$} \\
\hline$F$ & 96485 & {$\left[\mathrm{As} / \mathrm{mol}^{2}\right]$} \\
\hline$i_{\text {corr }}$ & $1 \mathrm{E}-06$ & {$\left[\mathrm{~A} / \mathrm{mm}^{2}\right]$} \\
\hline$C A R_{0}$ & 0.14 & {$[\mathrm{~mm}]$} \\
\hline$C A R_{M A X}$ & 0.28 & {$[\mathrm{~mm}]$} \\
\hline$\eta_{\text {lin }}$ & 0.7 & {$[-]$} \\
\hline$w / c$ & 0.5 & {$[-]$} \\
\hline$E_{c}$ & 32 & {$[\mathrm{GPa}]$} \\
\hline$f_{c t}$ & 4.5 & {$[\mathrm{MPa}]$} \\
\hline$E_{s}$ & 210 & {$[\mathrm{GPa}]$} \\
\hline$\mu_{s}$ & 0.3 & {$[-]$} \\
\hline$E_{c p}$ & 2.1 & {$[\mathrm{GPa}]$} \\
\hline$\mu_{c p}$ & 0.3 & {$[-]$} \\
\hline$V_{c p, \min }$ & 7.24 & {$\left[\mathrm{~mm}^{3}\right]$} \\
\hline$V_{c p, \max }$ & 38.52 & {$\left[\mathrm{~mm}^{3}\right]$} \\
\hline$n$ & 1.3 & {$[-]$} \\
\hline$\alpha$ & 1 & {$[1 / \mathrm{K}]$} \\
\hline$\chi$ & 0.65 & {$[-]$} \\
\hline & &
\end{tabular}

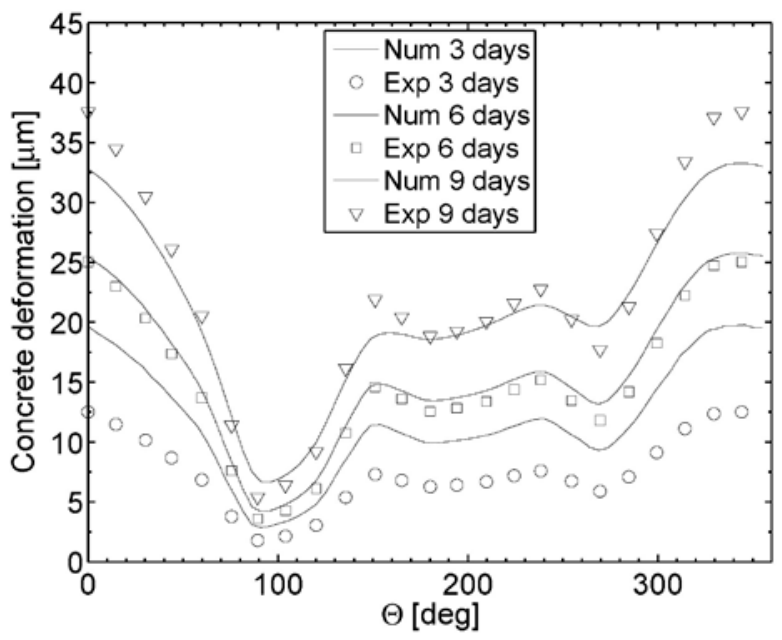

Figure 9: Modelled and experimentally observed (DIC measurements) corrosion-induced deformations along the circumference of the reinforcement.
As can be seen from the presented results in Fig. 9, good agreement (with respect to shape and magnitude) between experimentally observed and numerically predicted corrosioninduced deformations is found for six and nine days of accelerated corrosion. Higher deviations are observed for three days of accelerated corrosion, which may be attributed to assumptions with respect to the penetration of corrosion products (see Eq. 22 - 29), as well as material properties related to the corrosion products, such as stiffness and Poisson ratio.

In addition, experimentally observed microcracks (observed using digital image correlation (DIC)) that are due to accelerated corrosion are illustrated in Fig. 10. DIC allows for identification of cracks as zones with localized strain, i.e. areas in red and light blue in Fig. 10. Three corrosion-induced microcracks (with crack widths between approximately two and ten $\mu \mathrm{m}$ ) and considerable damage along the circumference of the reinforcement can be identified in Fig. 10. The microcracks formed at the reinforcement, extend around 10 to 15 $\mathrm{mm}$ into the mortar, and propagated towards the surface over time. For comparison, the crack pattern obtained by the presented modelling approach is given in Fig. 11. Similar crack pattern and corrosion-induced damage along the circumference of the reinforcement are obtained with the model. Extensive damage along the circumference of the reinforcement, in particular on the right side, and microcracking are observed.

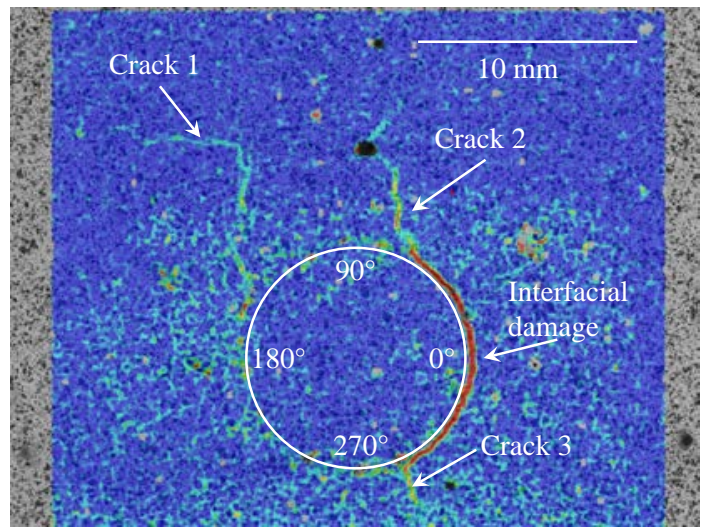

Figure 10: Corrosion-induced cracks in reinforced specimens after 9 days of accelerated corrosion, please note: only part of the test specimen is illustrated. 


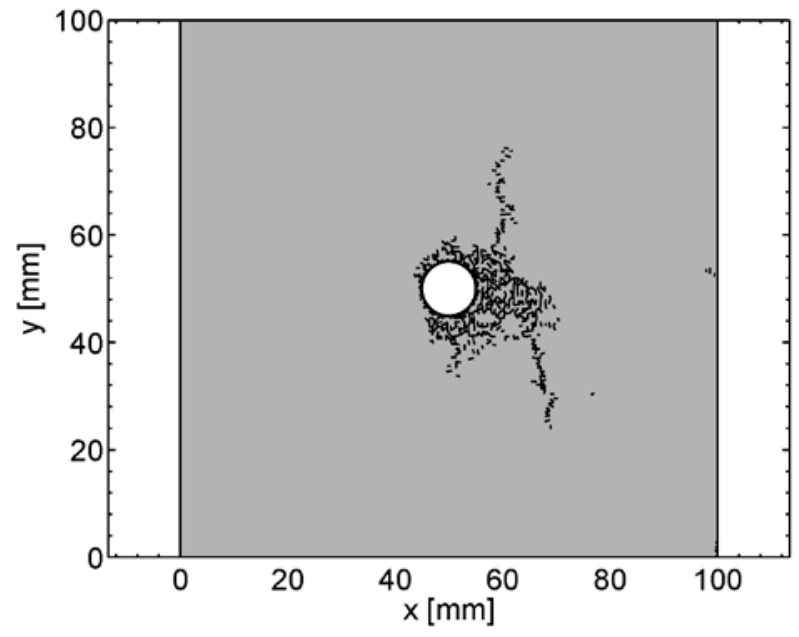

Figure 11: Modelled corrosion-induced crack pattern in test specimens.

Further, three microcracks can be observed in Fig. 11, which formed at the reinforcement and propagate towards the surface. While the direction of the microcracks is not completely in agreement with the experimental observations, the extent of the microcracks is in excellent agreement. The microcracks extend around 10 to $20 \mathrm{~mm}$ into the mortar.

To further demonstrate the applicability of the coupled lattice and FEM model, numerical simulations are compared to experimental data presented in [24]. $\mathrm{Vu}$ et al. [24] monitored corrosion-induced crack formation in reinforced concrete slabs $\left(250 \times 700 \times 1000 \mathrm{~mm}^{3}\right.$, i.e. $\mathrm{H} \times \mathrm{W} \times \mathrm{T}$ ) under accelerated conditions, i.e. a constant current was applied to polarize the steel and enforce active corrosion. Throughout the experiments, a constant corrosion current density of $100 \mu \mathrm{A} / \mathrm{cm}^{2}$ was applied. Assuming $100 \%$ current efficiency, i.e. all current applied is used for the formation of corrosion products, and uniform corrosion, Eq. 1 yields the cross sectional reduction of the reinforcement. The same input parameters (see Table 1) as for the previous example are used to simulate the experimental observations presented in [24]. However, Young's modulus (42.25 GPa) and tensile strength of concrete (3.94 MPa) as well as volume expansion coefficient of corrosion products (2.94) have been adopted from [24] for the simulation. In total, 364,428 DOFs were used to discretize the concrete, corrosion product, and reinforcement domain. While the concrete domain was discretized with 543,372 lattice elements, the corrosion product and reinforcement domain were discretized with 100 and 1078 elements, respectively. The minimum and maximum length of the lattice elements in the concrete domain were approximately 10 and $800 \mu \mathrm{m}$, respectively. Due to symmetry, and to save computational time, only a part of the slab was simulated.

Typical experimentally observed crack patterns after several weeks of accelerated corrosion are illustrated in Fig. 12. Apart from the main cracks that generally propagated vertically above the reinforcing bars, additional radial cracks were observed. The radial cracks had lengths between 80 and $150 \mathrm{~mm}$ and were inclined either 45 or 90 degrees to the main crack. For comparison, results of the presented modelling approach are illustrated in Fig. 13. As can be seen from the results, excellent agreement between experimentally observed and numerically predicted crack patterns can be observed with respect to orientation and location of predicted cracks.

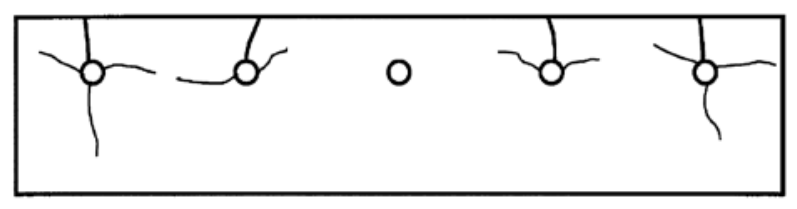

Figure 12: Typical crack patterns in test specimens subjected to accelerated corrosion, from [24].

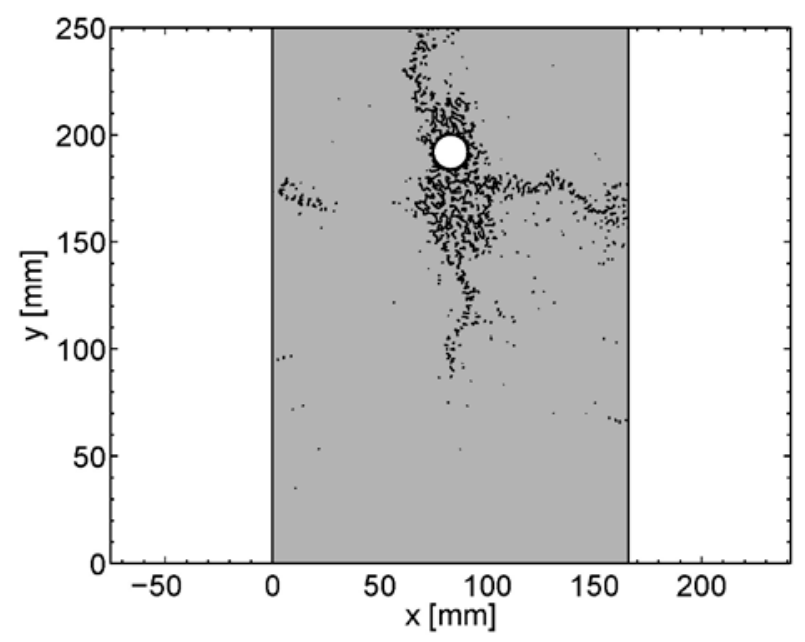

Figure 13: Predicted crack patterns in reinforced test specimens from [24] subjected to accelerated corrosion. 


\section{SUMMARY AND CONCLUSIONS}

A modelling approach for corrosioninduced damage in quasi-brittle materials was presented. The outlined modelling approach is embedded in an overarching cross disciplinary numerical framework that combines mechanical, chemical, and electrochemical processes on different length scales in reinforced concrete. In this paper, focus was placed on corrosion-induced damage processes, which were investigated by means of numerical simulations utilizing a coupled lattice and finite element method (FEM) modelling approach. While the reinforcement and surrounding corrosion product domain were discretized by continuum elements, a lattice approach was used for the discretization of the concrete domain. A thermal analogy was used to model the expansive nature of solid corrosion products and to describe corrosion-induced damages. The modelling approach accounts for the penetration of solid corrosion products into the available pore space of the surrounding cementitious materials, as well as non-uniform distribution of corrosion products along the circumference of the reinforcement.

To demonstrate the applicability of the presented modelling approach, numerical predictions in terms of corrosion-induced deformations, as well as formation and propagation of micro- and macrocracks were compared to experimental data obtained by digital image correlation and published in the literature. Excellent agreements between experimentally observed and numerically predicted crack patterns at the micro and macro scale indicate the capability of the modelling approach to accurately capture corrosion-induced damage phenomena in reinforced concrete. Moreover, good agreements were also found between experimental and numerical data for corrosioninduced deformations along the circumference of the reinforcement. Despite the good results found in the present study, future investigations should focus on more advances lattice element formulations, e.g. beam elements, as well as mechanical properties of the corrosion products formed and the penetration of those into the available pore space.

\section{ACKNOWLEDGMENTS}

Financial contributions from the Danish Expert Centre for Infrastructure Constructions, COWIfonden, and the project 'Sustainable Rehabilitation of Civil and Building Structures' funded by Nordic Innovation Centre, Project No. 08190 SR are greatly appreciated. This material is partially based upon work supported by the US National Science Foundation under Grant No. 1453881. Any opinions, findings, and conclusions or recommendations expressed in this material are those of the authors and do not necessarily reflect the views of the US NSF.

\section{REFERENCES}

[1] Michel, A., Stang, H., Lepech, M., and Geiker, M.R. 2015. Multi-physics and multi-scale deterioration modelling of reinforced concrete. Key Engineering Materials 665:13-16.

[2] Flint, M.M., Michel, A., Billington, S.L., and Geiker, M.R. 2014. Influence of Temporal Resolution of Boundary Conditions on the Moisture, Temperature and Ion Distributions in Concrete. Materials and Structures 47(4):729-748.

[3] Geiker, M., Nielsen, E.P., and Herfort, D. 2007. Prediction of chloride ingress and binding in cement paste. Materials and Structures 40:405-417.

[4] Michel, A., Geiker, M.R., Stang, H., and Lepech, M. 2015. Multi-physical and multi-scale deterioration modelling of reinforced concrete: coupling transport and corrosion at the material scale. In Stang, H. and Braestrup, M. (Eds) fib symposium proceedings.

[5] Lepech, M., Rao, A., Kiremidjian, A., Michel, A., Stang, H., and Geiker, M. 2015. Multi-physical and multi-scale deterioration modelling of reinforced concrete: coupling corrosion and damage for sustainability at the structural scale In Stang, H. and Braestrup, M. (Eds) fib symposium proceedings.

[6] Küter, A. 2009. Management of reinforcement corrosion: a thermodynamical approach. Ph.D. Thesis. Technical Uni- 
versity of Denmark, Kgs. Lyngby, Denmark.

[7] Koleva, D.A., Hu, J., Fraaij, A.L.A., Stroeven, P., Boshkov, N., and de Wit, J.H.W. 2006. Quantitative characterisation of steel/cement paste interface microstructure and corrosion phenomena in mortars suffering from chloride attack. Corrosion Science 48(12):4001-4019.

[8] Alonso, C., Andrade, C., Rodriguez, J., and Diez, J.M. 1998. Factors controlling cracking of concrete affected by reinforcement corrosion. Materials and Structures 31(211):435-441.

[9] Michel, A., Solgaard, A.O.S., Geiker, M., Stang, H. and Olesen, J.F. 2010. Modeling Formation of Cracks in Concrete Cover due to Reinforcement Corrosion. In Oh, B.H., Choi, O.C. and Chung, L. (Eds) Proceedings of FraMCoS-7; pp.944-951.

[10]Ngo, D. and Scordelis, A.C. 1967. Finite Element Analysis of Reinforced Concrete Beams. Journal of American Concrete Institute 64:152-163.

[11]Rashid, Y.R. 1968. Ultimate strength analysis of prestressed concrete pressure vessels. Nuclear Engineering and Design 7(4):334-344.

[12]Bolander, J.E. and Sukumar, N. 2005. Irregular lattice model for quasistatic crack propagation. Physical Review $B$ 71(9).

[13] Schlangen, E. 1993. Experimental and Numerical Analysis of Fracture Processes in Concrete. Ph.D. Thesis. Technical University of Delft, Delft, Netherlands.

[14]Qian, Z. 2012. Multiscale Modelling of Fracture Processes in Cementitious Materials. Ph.D. Thesis. Technical University of Delft, Delft, Netherlands.

[15] Herrmann, H.J., Hansen, H., and Roux, S. 1989. Fracture of disordered, elastic lattices in two dimensions, Physical Review B 39(1):637-648.

[16]Bazant, Z.P., Tabbara, M.R., Kazemi, M.T., and Pijaudier-Cabot, G. 1990. Random Particle Model for Fracture of Aggregate or Fiber Composites. Journal of Engineering Mechanics 116:1686-1705.

[17]Liu, Y. and Weyers, R. (1998). Modeling the time-to-corrosion cracking in chloride contaminated reinforced concrete, ACI Materials Journal 95(6):675.681.

[18]Caré, S., Nguyen, Q., L'Hostis, V., and Berthaud, Y. (2008). Mechanical properties of the rust layer induced by impressed current method in reinforced mortar, $\mathrm{Ce}$ ment and Concrete Research 38(8-9): 1079-1091.

[19] Val, D.V., Chernin, L. and Stewart, M.G. (2009). Experimental and numerical investigation of corrosion-induced cover cracking in reinforced concrete structures, Journal of Structural Engineering 135:376-385.

[20]Michel, A. Pease, B.J., Geiker, M.R., Stang, H., and Olesen, J.F. (2011). Monitoring reinforcement corrosion and corrosion-induced cracking using nondestructive $\mathrm{x}$-ray attenuation measurements. Cement and Concrete Research 41:1085-1094.

[21] Michel, A., Pease, B.J., Peterová, A., Geiker, M.R., Stang, S., and Thybo, A.E.A. (2014). Penetration of corrosion products and corrosion-induced cracking in reinforced cementitious materials: Experimental investigations and numerical simulations. Cement \& Concrete Composites 47:75-86.

[22] Powers, T. and Brownyard, T. (1947) Studies of the physical properties of hardened Portland cement paste. Journal of ACI 43(9):933-92.

[23]Pease, B.J., Michel, A., Thybo, A.E.A., and Stang, H. (2012). Estimation of elastic modulus of reinforcement corrosion products using inverse analysis of digital image correlation measurements for input in corrosion-induced cracking model. In Biondini, F. and Frangopol, D.M. (Eds), Bridge Maintenance, Safety, Management, Resilience and Sustainability. Proceedings of the 6th International Conference on Bridge Maintenance, Safety and Management (IABMAS 2012): pp.3643-3650.

[24] Vu K, Stewart M.G., and Mullard J. (2005). Corrosion-induced cracking: experimental data and predictive models. ACI Structural Journal 102(5):719-726. 